\title{
Healthcare Quality for Children and Adolescents with Suicidality Admitted to Acute Care Hospitals in the United States
}

\author{
JoAnna K. Leyenaar, MD, PhD, MPH*, Jennifer L. McLaren, MD²
}

\begin{abstract}
'Department of Pediatrics and The Dartmouth Institute For Health Policy and Clinical Practice, Geisel School of Medicine at Dartmouth, Lebanon, New Hampshire; ${ }^{2}$ Department of Psychiatry and The Dartmouth Institute for Health Policy and Clinical Practice, Geisel School of Medicine at Dartmouth, Lebanon, New Hampshire.
\end{abstract}

uicide is the second most common cause of death among children, adolescents, and young adults in the United States. In 2016, over 6,000 children and youth 5 to 24 years of age succumbed to suicide, thus reflecting a mortality rate nearly three times higher than deaths from malignancies and 28 times higher than deaths from sepsis in this age group. ' Suicidal ideation and suicide attempts are even more common, with $17 \%$ of high school students reporting seriously considering suicide and $8 \%$ reporting suicide attempts in the previous 12 months. ${ }^{2}$ These tragic statistics are reflected in our health system use, emergency department (ED) utilization for suicide attempts and suicidal ideation is growing at a tremendous rate, and over $50 \%$ of the children seen in EDs are subsequently admitted to the hospital for ongoing care. ${ }^{3,4}$

In this issue of Journal of Hospital Medicine, Doupnik and colleagues present an analysis of pediatric hospitalizations for suicide attempts and suicidal ideation at acute care hospitals contained within the 2013 and 2014 National Readmissions Dataset. ${ }^{5}$ This dataset reflects a nationally representative sample of pediatric hospitalizations, weighted to allow for national estimates. Although their focus was on hospital readmission, their analysis yielded additional valuable data about suicide attempts and suicidal ideation in American youth. The investigators identified 181,575 pediatric acute care hospitalizations for suicide attempts and suicidal ideation over the two-year study period, accounting for $9.5 \%$ of all acute care hospitalizations among children and adolescents 6 to 17 years of age nationally. This number exceeds the biennial number of pediatric hospitalizations for cellulitis, dehydration, and urinary tract infections, all of which are generally considered the "bread and butter" of pediatric hospital medicine. ${ }^{6}$

Doupnik and colleagues rightly pointed out that hospital readmission is not a nationally endorsed measure to evaluate the quality of pediatric mental health hospitalizations. At the same time, their work highlights that acute care hospitals need strategies to measure the quality of pediatric hospitalizations for suicide attempts and suicidal ideation. Beyond readmissions,

*Address for correspondence: Dr. JoAnna Leyenaar, Department of Pediatrics \& The Dartmouth Institute for Health Policy and Clinical Practice, Dartmouth-Hitchcock Medical Center, 1 Medical Center Way, Lebanon, NH, 03766; Telephone: 603-653-0855; E-mail: JoAnna.K.Leyenaar@hitchcock.org

Received: July 24, 2018; Accepted: August 29, 2018

๑) 2018 Society of Hospital Medicine DOI 10.12788/jhm.3092 how should the quality of these hospital stays be evaluated? A recent review of 15 national quality measure sets identified 257 unique measures to evaluate pediatric quality of care. ${ }^{7}$ Of these, only one focused on mental health hospitalization. This measure, which was endorsed by the National Quality Forum, determines the percentage of discharges for patients six years of age and older who were hospitalized for mental health diagnoses and who had a follow-up visit with a mental health practitioner within seven and 30 days of hospital discharge. ${ }^{8}$ Given Doupnik et al.'s finding that one-third of all 30-day hospital readmissions occurred within seven days of hospital discharge, early follow-up visits with mental health practitioners is arguably essential.

Although evidence-based quality measures to evaluate hospital-based mental healthcare are limited, quality measure development is ongoing, facilitated by recent federal health policy and associated research efforts. Four newly developed measures focus on the quality of inpatient care for suicidality, including two evaluated using data from health records and two derived from caregiver surveys. The first medical records-based measure identifies whether caregivers of patients admitted to hospital for dangerous self-harm or suicidality have documentation that they were counseled on how to restrict their child's or adolescent's access to potentially lethal means of suicide before discharge. The second record-based measure evaluates documentation in the medical record of discussion between the hospital provider and the patient's outpatient provider regarding the plan for follow-up. ${ }^{9}$ The two survey-based measures ask caregivers whether they were counseled on how to restrict access to potentially lethal means of suicide, and, for children and adolescents started on a new antidepressant medication or dose, whether they were counseled regarding the potential benefits and risks of the medication. ${ }^{10}$ All measures were field-tested at children's hospitals to ensure feasibility in data collection. However, as shown by Doupnik et al., only $7.4 \%$ of acute care hospitalizations for suicide attempts and suicidal ideation occurred at freestanding children's hospitals; most occurred at urban nonteaching centers. Evaluation of these new quality measures across structurally diverse hospitals is an important next step.

Beyond the healthcare constructs evaluated by these quality measures, many foundational questions about what constitutes high quality inpatient healthcare for suicide attempts and suicidal ideation remain. An American Academy of Child and Adolescent Psychiatry (AACAP) practice parameter, which was published in 2001, established minimal standards for the assessment and treatment of children and adolescents with suicidal behavior. ${ }^{11}$ 
This guideline recommends inpatient treatment until the mental state or level of suicidality has stabilized, with discharge considered only when the clinician is satisfied that adequate supervision and support will be available and when a responsible adult has agreed to secure or dispose of potentially lethal medications and firearms. It further recommends that the clinician treating the child or adolescent during the days following a suicide attempt be available to the patient and family_for example, to receive and make telephone calls outside of regular clinic hours. Recognizing the growing prevalence of suicidality in American children and youth, coupled with critical shortages in pediatric psychiatrists and fragmentation of inpatient and outpatient care, these minimal standards may be difficult to implement across the many settings where children receive their mental healthcare. $4,12,13$

The large number of children and adolescents being hospitalized for suicide attempts and suicidal ideation at acute care hospitals demands that we take stock of how we manage this vulnerable population. Although Doupnik and colleagues suggest that exclusion of specialty psychiatric hospitals from their dataset is a limitation, their presentation of suicide attempts and suicidal ideation epidemiology at acute care hospitals provides valuable data for pediatric hospitalists. Given the presence of pediatric hospitalists at many acute care hospitals, comanagement by hospital medicine and psychiatry services may prove both efficient and effective while breaking down the silos that traditionally separate these specialties. Alternatively, extending the role of collaborative care teams, which are increasingly embedded in pediatric primary care, into inpatient settings may enable continuity of care and improve healthcare quality. ${ }^{14}$ Finally, nearly 20 years have passed since the AACAP published its practice parameter for the assessment and treatment of children and adolescents with suicidal behavior. An update to reflect contemporary suicide attempts and suicidal ideation statistics and evidence-based practices is needed, and collaboration between professional pediatric and psychiatric organizations in the creation of this update would recognize the growing role of pediatricians, including hospitalists, in the provision of mental healthcare for children.

Updated guidelines must take into account the transitions of care experienced by children and adolescents throughout their hospital stay: at admission, at discharge, and during their hospitalization if they move from medical to psychiatric care. Research is needed to determine what proportion of children and adolescents receive evidence-based mental health therapies while in hospital and how many are connected with wraparound mental health services before hospital discharge. ${ }^{15}$ Doupnik et al. excluded children and adolescents who were transferred to other hospitals, which included over 18,000 youth. How long did these patients spend "boarding," and did they receive any mental health assessment or treatment during this period? Although the Joint Commission recommends that holding times for patients awaiting bed placement should not exceed four hours, hospitals have described average pediatric inpatient boarding times of two to three days while awaiting inpatient psychiatric care. ${ }^{16,17}$ In one study of children and adolescents awaiting transfer for inpatient psychiatric care, mental health counseling was received by only $6 \%$, which reflects lost time that could have been spent treating this highly vulnerable population. ${ }^{16}$ Multidisciplinary collaboration is needed to address these issues and inform best practices.

Although mortality is a rare outcome for most conditions we treat in pediatric hospital medicine, mortality following suicide attempts is all too common. The data presented by Doupnik and colleagues provide a powerful call to improve healthcare quality across the diverse settings where children with suicidality receive their care.

Disclosures: The authors have no financial relationships relevant to this article to disclose.

Funding: Dr. Leyenaar was supported by grant number K08HS024133 from the Agency for Healthcare Research and Quality. The content is solely the responsibility of the authors and does not necessarily represent the official views of $\mathrm{AHRQ}$

\section{References}

1. Centers for Disease Control and Prevention, National Center for Health Statistics. Underlying Cause of Death 1999-2016 on CDC WONDER Online Database, released December, 2017

2. Kann L, Kinchen S, Shanklin S, et al. Youth risk behavior surveillance-United States, 2013. MMWR. 2014;63(4):1-168.

3. Olfson M, Gameroff MJ, Marcus SC, Greenberg T, Shaffer D. Emergency treatment of young people following deliberate self-harm. Arch Gen Psychiatry. 2005;62(10):1122-1128. doi: 10.1001/archpsyc.62.10.1122

4. Mercado MC, Holland K, Leemis RW, Stone DM, Wang J. Trends in emergency department visits for nonfatal self-inflicted injuries among youth aged 10 to 24 years in the United States, 2001-2015. JAMA. 2017;318(19):1931-1932. doi: 10.1001/jama.2017.13317

5. Doupnik S, Rodean J, Zima B, et al. Readmissions after pediatric hospitalization for suicide ideation and suicide attempt. J Hosp Med. 2018;13(11):743751. doi: 10.12788/jhm.3070

6. Leyenaar JK, Ralston SL, Shieh M, Pekow PS, Mangione-Smith R, Lindenauer PK. Epidemiology of pediatric hospitalizations at general hospitals and freestanding children's hospitals in the United States. J Hosp Med. 2016;11(11):743-749. doi: 10.1002/jhm.2624

7. House SA, Coon ER, Schroeder AR, Ralston SL. Categorization of national pediatric quality measures. Pediatrics. 2017;139(4):e20163269.

8. National Quality Forum. Follow-up after hospitalization for mental illness. Available at www.qualityforum.org. Accessed July 21, 2018.

9. Bardach N, Burkhart Q, Richardson L, et al. Hospital-based quality measures for pediatric mental health care. Pediatrics. 2018;141(6):e20173554.

10. Parast L, Bardach N, Burkhart $Q$, et al. Development of new quality measures for hospital-based care of suicidal youth. Acad Pediatr. 2018;18(3):248-255. doi: 10.1016/j.acap.2017.09.017

11. Shaffer D, Pfeffer C. Practice parameters for the assessment and treatment of children and adolescents with suicidal behavior. J Am Acad Child Adolesc Psychiatry. 2001;40(7 Suppl):24-51. doi: 10.1097/00004583-200107001-00003

12. Thomas $C$, Holtzer $C$. The continuing shortage of child and adolescent psychiatrists. J Am Acad Child Adolesc Psychiatry. 2006;45(9):1023-1031. doi: 10.1097/01.chi.0000225353.16831.5d

13. Plemmons G, Hall M, Doupnik S, et al. Hospitalization for suicide ideation or attempt: 2008-2015. Pediatrics. 2018;141(6):e20172426.

14. Beach SR, Walker J, Celano CM, Mastromauro CA, Sharpe M, Huffman JC. Implementing collaborative care programs for psychiatric disorders in medical settings: a practical guide. Gen Hosp Psychiatry. 2015;37(6):522-527. doi: 10.1016/j.genhosppsych.2015.06.015

15. Winters N, Pumariega A. Practice parameter on child and adolescent mental health care in community systems of care. J Am Acad Child Adolsc Psychiatry. 2007;46(2):284-299. doi: 10.1097/01.chi.0000246061.70330.b8

16. Claudius I, Donofrio J, Lam CN, Santillanes G. Impact of boarding pediatric psychiatric patients on a medical ward. Hosp Pediatr. 2014;4(3):125-131. doi: 10.1542/hpeds.2013-0079

17. Gallagher KAS, Bujoreanu IS, Cheung P, Choi C, Golden S, Brodziak K. Psychiatric boarding in the pediatric inpatient medical setting: a retrospective analysis. Hosp Pediatr. 2013;7(8):444-450. doi: 10.1542/hpeds.2017-0005 